\title{
Drug-DNA Interaction: A Theoretical Study of the Stability of CP-DNA Binding with Thionine
}

\author{
Ghazala Yunus*, Seema Srivastava, Vishwambhar Dayal Gupta \\ Department of Physics, Integral University, Lucknow, India \\ Email:"ghazala_kuddus@yahoo.com
}

Received March 24, 2012; revised April 22, 2012; accepted May 9, 2012

\begin{abstract}
The recent study on binding of small molecules to double stranded DNA suggested that the intercalation of a tricyclic heteroaromatic molecule, thionine, with natural DNA provided thermal stabilization to the complex. In the present study, we reported theoretical analysis of thionine binding with Clostridium perfringenes DNA duplex (CP-DNA) by using an amended Zimm and Bragg theory, to explain the melting behaviour and heat capacity of CP-DNA with and without thionine binding. The experimental models of Paul et al. (2010) have been used for the study. The sharpness of transition has been examined in terms of half width and sensitivity parameter $(\Delta \mathrm{H} / \sigma)$. The results of theoretical approach suggested that the various parameters such as transition profile, sharpness of the transition, heat capacity curve and half widths are in good agreement with the experimental measurements for binding of thionine. Therefore, the proposed theoretical analysis may be useful in order to understand interaction of small molecules to DNA that may be applied in the process of drug development and for designing more potential DNA binding therapeutic molecules.
\end{abstract}

Keywords: Thionine; DNA Binding; Transition Profile; Heat Capacity; Drug Design

\section{Introduction}

The study of the interaction of small molecules with DNA is a field of high tropical interest and the number and verity of techniques devoted to evidence drug-DNA interactions is continuously growing. The binding of heterocyclic aromatic molecules to nucleic acids has received considerable attention over the past several years due to their relevance in various biological applications including cancer chemotherapy [1-8]. Accordingly, a number of experimental studies are conducted to understand the nature and thermodynamics of heterocyclic aromatic molecules and nucleic acid interaction [7,9-16]. Thionine (3,7-Diamino-5-phenothiazinium) (Figure 1), a tricyclic heteroaromatic molecule, has been studied for its intercalative interaction with duplex DNA [13] and photoinduced mutagenic actions on binding to DNA [11]. On the basis of satellite hole spectroscopy study Chang et al. [17] recommended that thionine binds specifically with guanine-cytosine (GC) contents of duplex DNA. However, Tuite and Kelly [13] suggested that (GC) specificity of thionine binding was not very marked. The model proposed by Hecht et al. $[18,19]$ suggested that thionine exists in two different tautomeric forms and the amino form is intercalated while the imino form is not. Although these data provide somewhat cont radicting

"Corresponding author. data on the GC specificity of thionine binding to linear double stranded DNA. A recent study [18] by pressure tuning hole burning spectroscopy has conclusively shown an external stacking mode of binding of thionine to quadruplex structures. Thus, although some structural data is available, the same is not conclusive and the thermodynamics of thionine binding to DNA is not yet elucidated. The calorimetric analysis of thionine binding to a DNA duplex was reported by Paul et al. [7]. They also investigated the structural and thermodynamic aspects of thionine binding to natural DNAs of varying base composition. They showed strong binding of thionine with CP-DNA which increases the thermal stability of the duplex, and at saturation the duplex melts with a Tm some $6.3^{\circ} \mathrm{C}$ above that of the free duplex [7]. They also concluded that binding of thionine to CP-DNA is an exothermic process and there is involvement of multiplicity of non covalent interactions in the binding process. In the present study, we have attempted to understand the effect of thionine binding on a native DNA duplex using the model of Paul et al. [7] who studied the thermal and<smiles>Nc1ccc2nc3ccc(N)cc3[s+]c2c1</smiles>

Figure 1. Chemical structure of thionine. 
thermodynamic behaviour of thionine binding to CP-DNA. To elucidate lambda point anomalies in heat capacities and order-disorder transition in thionine bounded and unbounded DNA duplexes, we used amended Zimm and Bragg theory which is considered initially for the helix coil transitions in polypeptides [20-22]. The effect of thionine binding is reflected in the change in nucleation parameter, which is an inverse measure of binding strength.

\section{Theory}

The results of the structural studies proposed by Paul et al. [7] for the complex of heterocyclic aromatic molecules with DNA on the basis of spectroscopic and calorimetric study, as mentioned above, suggested that thionine binds strongly with the Clostridium perfringenes DNA that resulted in thermal stabilization of the complex. The binding of thionine to the AT rich Clostridium perfringenes DNA was intercalative and favored by higher entropic contribution indicating the stronger perturbation of the water structure associated with the AT sequences [7]. However, the system remains a highly co-operative one therefore the co-operative transition theory could be applied to explain the melting profile and temperature dependence of thermodynamical parameter, such as heat capacity. Therefore, we can use amended Zimm and Bragg theory [20] which is described in our previous publication [23]. Briefly, the above mentioned theory consists of writing an Ising matrix for a two-phase system, the bounded state and unbounded state. As discussed earlier [21,22,24-26] and by Zimm and Bragg [20], the Ising matrix $M$ can be written as:

$$
\begin{aligned}
& f_{r} \quad f_{k} \quad f_{h} \\
& f_{r} \mid f_{r}^{1 / 2} f_{r}^{1 / 2} \quad f_{r}^{1 / 2} f_{k}^{1 / 2} \quad 0 \\
& \boldsymbol{M}=f_{k} f_{r}^{1 / 2} f_{k}^{1 / 2} \quad 0 \quad f_{k}^{1 / 2} f_{h}^{1 / 2} \\
& f_{h}\left|f_{h}^{1 / 2} f_{r}^{1 / 2} \quad 0 \quad f_{h}^{1 / 2} f_{h}^{1 / 2}\right|
\end{aligned}
$$

where $f_{\mathrm{r}}, f_{\mathrm{h}}$ and $f_{\mathrm{k}}$ are corresponding base pair partition functions' contributions in the three states i.e. ordered, disordered and boundary or nucleation. The eigen values of $\boldsymbol{M}$ are given by:

$$
\begin{aligned}
& \lambda_{1}=\left[\left(f_{r}+f_{h}\right)+\left\{\left(f_{r}-f_{h}\right)^{2}+4 f_{r} f_{k}\right\}^{1 / 2}\right] \\
& \lambda_{2}=\left[\left(f_{r}+f_{h}\right)-\left\{\left(f_{r}-f_{h}\right)^{2}+4 f_{r} f_{k}\right\}^{1 / 2}\right] \\
& \lambda_{3}=0
\end{aligned}
$$

Since we are dealing with a finite system hence the effect of initial and final states becomes important. The contribution of the first segment to the partition function is given by:

$$
\boldsymbol{U}=\left(f_{r}^{1 / 2}, 0,0\right)
$$

where the column vector $\boldsymbol{V}$ gives the state of the last segment,

$$
\boldsymbol{V}=\left|\begin{array}{c}
f_{r}^{1 / 2} \\
f_{k}^{1 / 2} \\
f_{h}^{1 / 2}
\end{array}\right|
$$

The partition function for an $N$-segment chain is given by;

$$
\boldsymbol{Z}=\boldsymbol{U} \boldsymbol{M}^{N-1} \boldsymbol{V}
$$

The matrix $\boldsymbol{T}$ which diagnolizes $\boldsymbol{M}$ consists of the column vectors given by:

$$
\boldsymbol{M X}=\lambda \boldsymbol{X}
$$

where, $\boldsymbol{X}=\left|\begin{array}{c}X_{1} \\ X_{2} \\ X_{3}\end{array}\right|$.

By substituting the values of $\boldsymbol{M}$ from Equation (6), we get:

$$
\boldsymbol{T}=\left|\begin{array}{ccc}
1 & 1 & 1 \\
\frac{\left(\lambda_{1}-f_{r}\right)}{\left(f_{r}^{1 / 2} f_{k}^{1 / 2}\right)} & \frac{\left(\lambda_{1}-f_{r}\right)}{\left(f_{r}^{1 / 2} f_{k}^{1 / 2}\right)}-\left(f_{r}^{1 / 2} f_{k}^{1 / 2}\right) \\
\frac{\left(f_{h}^{1 / 2} f_{r}^{1 / 2}\right)}{\left(\lambda_{1}-f_{h}\right)} & \frac{\left(f_{h}^{1 / 2} f_{r}^{1 / 2}\right)}{\left(\lambda_{1}-f_{h}\right)}-\left(f_{h}^{1 / 2} f_{r}^{1 / 2}\right)
\end{array}\right|
$$

Similarly, we get $\boldsymbol{T}^{-1}$ from the matrix equation

$$
\boldsymbol{Y M}=\lambda \boldsymbol{Y}
$$

where, $\boldsymbol{Y}=\left[Y_{1} Y_{2,} Y_{3}\right]$

Again by substituting the values of $\boldsymbol{M}$ from Equation (1) in Equation (8), we get:

$$
\boldsymbol{T}^{1}=\left|\begin{array}{ccc}
C_{1} & \frac{c_{1}\left(f_{r}^{1 / 2} f_{k}^{1 / 2}\right)}{\lambda_{1}} & \frac{c_{1}\left(f_{k} f_{r}^{1 / 2} f_{h}^{1 / 2}\right)}{\lambda_{1}\left(\lambda_{1}-f_{h}\right)} \\
C_{2} & \frac{c_{2}\left(f_{r}^{1 / 2} f_{k}^{1 / 2}\right)}{\lambda_{2}} & \frac{c_{2}\left(f_{k} f_{r}^{1 / 2} f_{h}^{1 / 2}\right)}{\lambda_{2}\left(\lambda_{2}-f_{h}\right)} \\
C_{3} & \frac{c_{3}\left(f_{r}^{1 / 2} f_{k}^{1 / 2}\right)}{\lambda_{3}} & \frac{c_{3}\left(f_{k} f_{r}^{1 / 2} f_{h}^{1 / 2}\right)}{\lambda_{3}\left(\lambda_{3}-f_{h}\right)}
\end{array}\right|
$$

The normalization constants are:

$$
C_{1}=\frac{\lambda_{1}-f_{h}}{\lambda_{1}-\lambda_{2}}, C_{2}=\frac{\lambda_{2}-f_{h}}{\lambda_{2}-\lambda_{1}}, C_{3}=0
$$

If we let $\Lambda=\boldsymbol{T}^{-1} \boldsymbol{M T}$ be the diagonalized form of $\boldsymbol{M}$, the partition function can be written as:

$$
\boldsymbol{Z}=\boldsymbol{U} \boldsymbol{T} \Lambda N^{-1} \boldsymbol{T}^{-1} \boldsymbol{V}
$$


On substituting the values from Equations (1), (3), (4), (7), (9) and (10) in Equation (11), the partition function becomes:

$$
\mathbf{Z}=C_{1} \lambda_{1}^{N}+C_{2} \lambda_{1}^{N}
$$

The fraction of the segments in the disordered form is given by

$$
Q_{r}=\left[\delta \ln Z / \delta \ln f_{r}\right] / N
$$

Solving the above equation, we get:

$$
Q_{r}=\frac{1}{2}+\frac{(1-s)(2 A-1)}{2 P}+\frac{(1+s)\{(2 A-1) P-1+s\}}{2 P^{2} N}
$$

$$
\begin{aligned}
& P=\frac{\lambda_{1}-\lambda_{2}}{f_{r}} \\
& s=\frac{f_{h}}{f_{r}} \\
& \sigma=\frac{f_{k}}{f_{r}} \\
& A=\left[\left(f_{r}-f_{h}\right)^{2}+4 f_{k} f_{r}\right]^{-2} \\
& \frac{\delta Q_{r}}{\delta S}=\left(\frac{1}{2 P^{2}}\right)\left[\frac{2 P(1-S) \delta A}{\delta S}-P(2 A-1)-\frac{(1-S)(2 A-1) \delta P}{\delta S}\right] \\
& \frac{\delta A}{\delta S}=\left\{\frac{(S-\sigma)^{N}}{\left(Z / f_{r}^{N}\right)^{2}}\right\} \times\left(\frac{\sigma}{P^{3}}\right) \times\left[-2+\left\{\frac{N(S-2 \sigma-1)}{S}-\sigma\right\}\right]
\end{aligned}
$$$$
+\left(\frac{1}{2 P^{3} N}\right)\left[P\left\{(S+1)\left\{\frac{(2 A-1) \delta P}{\delta S}+\frac{2 P \delta A}{\delta S}+1\right\}+\{(2 A-1) P-1+S\}-\{(2 A-1) P-1+S\} 2(S+1)\right\}\right]
$$

here $s$ is propagation parameter, which for simplicity is assumed to be 1 . In fact, in most of the systems, it is found to be close to unity. If $A_{r}$ and $A_{h}$ are the absorbance in disordered and ordered states, respectively, the total absorption can be written as:

$$
A=Q_{r} A_{r}+\left(1-Q_{r}\right) A_{h}
$$

The extension of this formalism to specific heat $\left(C_{v}\right)$ is straight forward. The specific heat is related to the molar enthalpy and entropy changes in the transition from state I to II. From the well known thermodynamic relations, free energy and internal energy are $F=-K T \ln Z$ and $U=-T^{2}(\delta / \delta T)(F / T)$, respectively. Differentiating internal energy with respect to temperature we get the specific heat:

$$
C_{v}=\delta U / \delta T=N_{k}\left(\Delta H / R T_{m}\right)^{2}\left(S \delta Q_{r} / \delta S\right)
$$

where $\Delta H$ is the molar change in enthalpy about the transition point, $S$ is entropy which is equal to

$$
S=\exp \left[(\Delta H / R)\left\{(1 / T)-\left(1 / T_{m}\right)\right\}\right]
$$

$T_{m}$ is the transition temperature, and $\delta P / \delta S=(S-1) / P$ and $\sigma=f_{k} / f_{r} ; \sigma$ is the nucleation parameter and is a measure of the energy expanded/released in the formation (uncoiling) of first turn of the ordered/disordered state. It is related to the uninterrupted sequence lengths [20]. The volume heat capacity $C_{v}$ has been converted into constant pressure heat capacity $C_{p}$ by using the Nernst-Lindemann approximation [26]:

$$
C_{p}-C_{v}=3 R A_{0}\left(C_{p}^{2} T / C_{v} T_{m}\right)
$$

where $A_{0}$ is a constant often of a universal value [3.9 $\times$ $\left.10^{-9}(\mathrm{Kmol}){ }^{\mathrm{J}-1}\right]$ and $T_{m}$ is the melting temperature.

\section{Results}

\subsection{Transition Profiles}

The binding affinity of thionine with C-DNA has been studied by spectroscopic, fluorescence and dialysis methods which showed strong intercalative binding and enabled the assumption of two state systems consisting of bound and free thionine [7]. When tricyclic heteroaromatic thionine binds with natural Clostridium perfringenes DNA duplex, the structure of DNA duplex still remains a very much co-operative and thus two-state theory of order-disorder transition is applicable. The Zimm and Bragg theory [7] is amended so as to consider ordered (bounded/unbounded) and disordered states as the two states which co-exist at the transition point. The transition is characterized mainly by the nucleation parameter and overall change of enthalpy/entropy, which are also the main thermodynamic forces driving the transition. The change in enthalpy obtained from differential scanning calorimetric measurements takes all this into account. This is evident from the enthalpy change and changes in other transition parameters, such as nucleation parameter $(\sigma)$ and melting point (Table 1). The melting temperature of the duplex was increased after binding with thionine at saturation. At saturation, the duplex melts with a $T_{m}$ some $6.5^{\circ} \mathrm{C}$ above that of the free duplex. The sharpness of the transition can be looked at in terms of half width and a sensitivity parameter defined as $(\Delta H / \sigma)$. The variation of these parameters systematically reflects that the transition is sharp in case of unbounded state and goes blunt with thionine saturation. In case of 
$\lambda$-transition, the same trend in the sharpness of transition is seen between the thionine bounded as well as unbounded curves. As expected, the sharpness is better in unbounded, as compared to bounded state. The various parameters, which give transition profiles in best agreement with the experimental measurements for binding of thionine to CP-DNA, are given in Table 1.

The transition profiles and heat capacity for unbounded DNA duplex and bounded with thionine are shown in Figure 2. Minor insignificant deviation at the tail ends is primarily due to the presence of various disordered states and presence of short helical segments found in the random coil states. Figure 2 A shows experimental and calculated transition curves for the duplex in the absence of thionine and B shows the transition when the duplex is saturated with thionine (thionine/ DNA $=0.4$ ). As expected, a cooperative transition profile is observed with calculated data. The conclusion deduced from the theoretical data is consistent with the directly measured binding enthalpy by Paul et al. [7] determined through DSC.

Table 1. Transition parameters for thionine binding to CPDNA.

\begin{tabular}{lll}
\hline Parameters & $\begin{array}{l}\text { Unbounded } \\
\text { CP-DNA }\end{array}$ & $\begin{array}{l}\text { CP-DNA saturated } \\
\text { with thionine }\end{array}$ \\
\hline $\mathrm{T}_{\mathrm{m}}(\mathrm{K})$ & 345 & 351.5 \\
$\Delta H \mathrm{Kcal} / M \mathrm{bp}$ & $3.06 \times 10^{3}$ & $3.24 \times 10^{3}$ \\
$\sigma$ & $3 \times 10^{-4}$ & $5 \times 10^{-4}$ \\
$N$ & 98 & 90 \\
$A_{h}$ & 0 & 0 \\
$A_{r}$ & 1 & 1 \\
Half width (Exp.) & 5 & 6.5 \\
Half width (Theo.) & 5 & 6.5 \\
Sensitivity parameter $(\Delta H / \sigma)$ & $102 \times 10^{5}$ & $648 \times 10^{4}$ \\
\hline
\end{tabular}

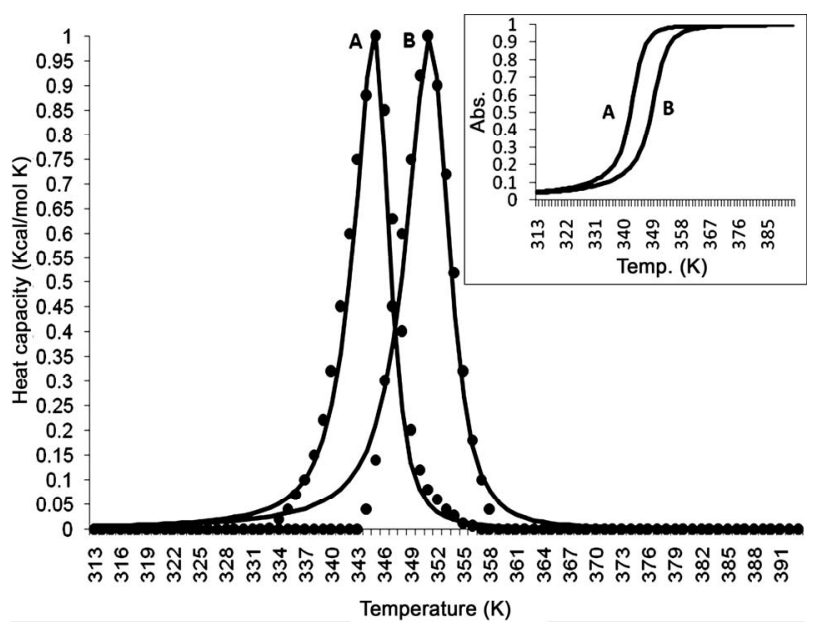

Figure 2. Heat capacity and transition profiles (inset) for thionine bounded and unbounded CP-DNA. A: Unbounded state; B: Bounded state at saturation. [(-) calculated and (•••) experimental values].

\subsection{Heat Capacity}

The conformational and dynamical states of a macromolecular system are characterized by heat capacity which is second derivative of the free energy and has been calculated by using Equation (14). These heat capacity curves with $\lambda$-point anomaly are shown in Figure $\mathbf{2}$ along with their transition profile. The theoretically obtained heat capacity profiles agreed with the experimentally reported ones and could be brought almost into coincidence with the use of scaling factors, which is very close to one in transition profiles and slightly higher for the heat capacity curves. The sharpness of the transition can be characterized by the half widths of the heat capacity curves that are in good agreement in both experimental and theoretical graphs.

\section{Discussion}

The present study concluded that the natural CP-DNA molecule is an extremely co-operative structure and when thionine bind to it the co-operativity is not so much disturbed. Therefore, the amended Zimm and Bragg theory (phase transitions theory) can be effectively applied to it. It generates the experimental transition profile and $\lambda$ point heat capacity anomaly successfully. These results will allow us to assess the thermodynamic profile of the binding process. Our theoretical studies of heterocyclic aromatic molecules binding are being extended to other synthetic and natural nucleic acids. The theoretical data obtained also demonstrated that the binding of thionine to $\mathrm{CP}-\mathrm{DN}$ is an endothermic process and that this binding increases the melting temperature of the duplex as supported by calorimetric measurement. The specific binding and intercalation of thionine with DNA have been studied by using spectroscopic methods [13,17]. However, Paul and her coworkers used optical melting and DSC techniques to understand the interaction of small molecule, thionine, with double stranded deoxyribonucleic acid, native CP-DNA [7]. The binding of thionine stabilized CP-DNA and $\Delta T_{m}$ value of about $6.3^{\circ} \mathrm{C}$ was obtained under saturating condition. Accordingly, we can interpret our theoretical results in the context of the specific structural features of the complex as deduced from their spectroscopic/DSC data. Inspection of Figures $2 \mathrm{~A}$ and $B$ reveals that the transition of the thionine-saturated CP-DNA duplex is significantly broader than the transition of the thionine-free duplex. Thus, in addition to affecting the transition enthalpy and the melting temperature, binding of heterocyclic aromatic molecules also alters the nature of the transition as reflected by the increase in transition width in experimental and calculated (theoretical) both data. In recent years, an increased understanding of the role played by nucleic acids in biological systems made DNA an alternative candidate for 
the development of new drugs. The successful applications of molecular modeling in virtual ligand screening and structure-based design of organic and inorganic molecules that target DNA are highlighted by Ma et al. [27]. The interactions of drugs and calf thymus DNA were also investigated by using non-linear fit analysis [28]. Other recent literatures also reviewed that the study of DNA interaction with a small molecules is of high interest [7,29-32]. Thus, the present theoretical analysis can be applied to understand bimolecular interaction and may also be implicated to the process of drug development in biomedical industries.

\section{Acknowledgements}

Technical assistance from Dr. Mohammed Kuddus, Department of Biotechnology, Integral University, Lucknow is gratefully acknowledged.

\section{REFERENCES}

[1] M. J. Waring, "DNA Modification and Cancer," Annual Reviews Biochemistry, Vol. 50, 1981, pp. 159-192. doi:10.1146/annurev.bi.50.070181.001111

[2] L. H. Hurley, "Secondary DNA Structures as Molecular Targets for Cancer Therapeutics," Biochemical Society Transactions, Vol. 29, No. 6, 2001, pp. 692-696.

[3] L. H. Hurley, "DNA and Its Associated Processes as Targets for Cancer Therapy," Nature Reviews Cancer, Vol. 2, No. 3, 2002, pp. 188-200. doi:10.1038/nrc749

[4] R. Martinez and L. Chacon-Garcia, "The Search of DNAIntercalators as Antitumoral Drugs: What It Worked and What Did Not Work," Current Medicinal Chemistry, Vol. 12, No. 2, 2005, pp. 127-151.

[5] R. Palchaudhuri and P. J. Hergenrother, "DNA as a Target for Anticancer Compounds: Methods to Determine the Mode of Binding and the Mechanism of Action," Current Opinion in Biotechnololy, Vol. 18, No. 6, 2007, pp. 497-503. doi:10.1016/j.copbio.2007.09.006

[6] M. Maiti and G. S. Kumar, "Molecular Aspects on the Interaction of Protoberberine, Benzophenanthridine, and Aristolochia Group of Alkaloids with Nucleic Acid Structures and Biological Perspectives," Medicinal Research Reviews, Vol. 27, No. 5, 2007, pp. 649-695. doi: $10.1002 /$ med.20087

[7] P. Paul, M. Hossain, R. C. Yadav and G. S. Kumar, "Biophysical Studies on the Base Specificity and Energetics of the DNA Interaction of Photoactive Dye Thionine: Spectroscopic and Calorimetric Approach," Biophysical Chemistry, Vol. 148, No. 1-3, 2010, pp. 93-103. doi:10.1016/j.bpc.2010.02.015

[8] K. Bhadra and G. S. Kumar, "Therapeutic Potential of Nucleic Acid-Binding Isoquinoline Alkaloids: Binding Aspects and Implications for Drug Design," Medicinal Research Reviews, Vol. 31, No. 6, 2011, pp. 821-862. doi:10.1002/med.20202

[9] J. Y. Du, X. H. Huang, F. Xu, Y. Y. Feng, W. Xing and T.
H. Lu, "Spectral Study on the Interaction Mechanism between Thionine and Calf Thymus DNA," Guang $P u$ Xue Yu Guang Pu Fen Xi, Vol. 25, No. 9, 2005, 14351448.

[10] S. Jockusch, D. Lee, N. J. Turro and E. F. Leonard, "PhotoInduced Inactivation of Viruses: Adsorption of Methylene Blue, Thionine, and Thiopyronine on Beta Bacteriophage," Proceeding of National Academy of Sciences USA, Vol. 93, No. 15, 1996, pp. 7446-7451.

doi:10.1073/pnas.93.15.7446

[11] C. Dohno, E. D. A. Stemp and J. K. Barton, "Fast Back Electron Transfer Prevents Guanine Damage by Photoexcited Thionine Bound to DNA" Journal of American Chemical Society, Vol. 125, No. 32, 2003, pp. 9586-9587. doi:10.1021/ja036397z

[12] Y. Xu, L. Yang, X. Ye, P. He and Y. Fang, "ImpedanceBased DNA Biosensor Employing Molecular Beacon DNA as Probe and Thionine as Charge Neutralizer," Electroanalysis, Vol. 18, No. 9, 2006, pp. 873-881. doi:10.1002/elan.200503465

[13] E. M. Tuite and J. M. Kelly, "The Interaction of Methylene Blue, Azure B, and Thionine with DNA: Formation of Complexes with Polynucleotides and Mononucleotides as Model Systems," Biopolymers, Vol. 35, No. 5, 1995, pp. 419-433. doi:10.1002/bip.360350502

[14] Y. J. Ihaya and T. Nakamura, "Optical Activity of Nucleic Acid-Thionine Complexes," Bulletin of the Chemical Society of Japan, Vol. 44, 1971, pp. 951-957.

[15] C. V. Kumar and E. H. Asuncion, "DNA Binding Studies and Site Selective Fluorescence Sensitization of an Anthryl Probe," Journal of American Chemical Society, Vol. 115, No. 19, 1993, pp. 8547-8553. doi: $10.1021 / \mathrm{ja} 00072 \mathrm{a} 004$

[16] E. M. Tuite and J. M. Kelly, "New Trends in Photobiology: Photochemical Interactions of Methylene Blue and Analogues with DNA and Other Biological Substrates," Journal of Photo chemistry and Photobiology, B: Biology, Vol. 21, No. 2-3, 1993, pp. 103-124. doi:10.1016/1011-1344(93)80173-7

[17] T. C. Chang, Y. P. Yang, K. H. Huang, C. C. Chang and C. Hecht, "Investigation of Thionine-DNA Interaction by Satellite Hole Spectroscopy," Optics and Spectroscopy, Vol. 98, No. 5, 2005, pp. 716-721. doi:10.1134/1.1929049

[18] C. Hecht, J. Friedrich and T. C. Chang, "Interactions of Thionin with DNA Strands: Intercalation versus External Stacking," Journal of Physical Chemistry B, Vol. 108, No. 29, 2004, pp. 10241-10244. doi:10.1021/jp0372993

[19] C. Hecht, P. Hermann, J. Friedrich and T. C. Chang, "Thionin in a Cyclodextrin Nanocavity: Measuring Local Compressibilities by Pressure Tuning Hole Burning Spectroscopy," Chemical Physics Letters, Vol. 413, No. 4-6, 2005, pp. 335-341. doi:10.1016/j.cplett.2005.08.002

[20] B. H. Zimm and J. K. Bragg, "Theory of the Phase Transition between Helix and Random Coil in Polypeptide Chains," Journal of Chemical Physics, Vol. 31, No. 2, 1959, pp. 526-535. doi:10.1063/1.1730390

[21] S. Srivastava, V. D. Gupta, P. Tandon, S. Singh and S. B. Katti, "Drug Binding and Order-Order and Order-Disor- 
der Transitions in DNA Triple Helices," Journal of Macromolecular Science Physics, Vol. 38, No. 4, 1999, pp. 349-366. doi:10.1080/00222349908212437

[22] S. Srivastava, S. Srivastava, S. Singh and V. D. Gupta, "Stability and Transition in a DNA Tetraplex: A Model for Telomeres," Journal of Macromolecular Science Physics, Vol. 40, No. 1, 2001, pp. 1-14. doi:10.1081/MB-100000050

[23] G. Yunus, S. Srivastava and V. D. Gupta, "Stability of DNA Binding with Dipyrandium: A Theoretical Study," International Journal of Physical Sciences, Vol. 6, No. 36, 2011, pp. 8151-8156. doi: 10.5897/IJPS11.1335

[24] S. Srivastava, I. A. Khan, S. Srivastava and V. D. Gupta, "A Theoretical Study of the Stability of DNA Binding with Cis/Trans Platin," Indian Journal of Biochemistry and Biophysics, Vol. 41, No. 6, 2004, pp. 305-310.

[25] N. Poklar, D. S. Pilch, S. J. Lippard, E. A. Redding, S. U. Dunham and K. J. Breslauer, "Influence of Cisplatin Intrastrand Crosslinking on the Conformation, Thermal Stability, and Energetics of a 20-mer DNA Duplex," Proceeding of National Academy of Sciences USA, Vol. 93, No. 15, 1996, pp. 7606-7611. doi:10.1073/pnas.93.15.7606

[26] K. A. Roles and B. Wunderlich, "Heat Capacities of Solid Poly(Amino Acids). I. Polyglycine, Poly(L-Alanine), and Poly(L-Valine)," Biopolymers, Vol. 31, No. 5, 1991, pp. 477-487. doi:10.1002/bip.360310503

[27] D. L. Ma, D. S. Chan, P. Lee, M. H. Kwan and C. H. Leung, "Molecular Modeling of Drug-DNA Interactions: Virtual Screening to Structure-Based Design," Biochimie,
Vol. 93, No. 8, 2011, pp. 1252-1266. doi:10.1016/j.biochi.2011.04.002

[28] J. Yuan, W. Guo and E. Wang, "Oligonucleotide Stabilized Silver Nanoclusters as Fluorescence Probe for DrugDNA Interaction Investigation," Analitica Chimica Acta, Vol. 706, No. 2, 2011, pp. 338-342.

doi:10.1016/j.aca.2011.08.043

[29] F. Araya, G. Huchet, I. McGroarty, G. G. Skellern and R. D. Waigh, "Capillary Electrophoresis for Studying DrugDNA Interactions," Methods, Vol. 42, No. 2, 2007, pp. 141-149. doi:10.1016/j.ymeth.2006.09.006

[30] M. M. Islam, S. R. Chowdhury and G. S. Kumar, "Spectroscopic and Calorimetric Studies on the Binding of Alkaloids Berberine, Palmatine and Coralyne to Double Stranded RNA Polynucleotides," Journal of Physical Chemistry B, Vol. 113, No. 4, 2009, pp. 1210-1224. doi:10.1021/jp806597w

[31] V. Gonzalez-Ruiz, A. I. Olives, M. A. Martin, P. Ribelles, M. T. Ramos and J. C. Menendez, "An Overview of Analytical Techniques Employed to Evidence Drug-DNA Interactions. Applications to the Design of Genosensors," In: M. A. Komorowska and S. Olsztynska-Janus, Eds., Biomedical Engineering, Trends, Research and Technologies, 2011, InTech, Castle Rock, pp. 65-90. doi:10.5772/13586

[32] A. Kunwar, S. Emmanuel, U. Singh, R. K. Chittela, D. Sharma, S. K. Sandur and I. K. Priyadarsini, "Interaction of a Curcumin Analogue Dimethoxycurcumin with DNA," Chemical Biology and Drug Design, Vol. 77, No. 4, 2011, pp. 281-287. doi:10.1111/j.1747-0285.2011.01083.x 